\title{
A Grey Seasonal Index Model for Forecasting Groundwater Depth of Ningxia Plain
}

\author{
Ziqi Yin $\mathbb{D}^{1}$ and Kai Zhang $\mathbb{D D}^{2,3}$ \\ ${ }^{1}$ Faculty of Information Technology, Macau University of Science and Technology, Macau, China \\ ${ }^{2}$ School of Management Science and Engineering, Shandong University of Finance and Economics, Shandong, China \\ ${ }^{3}$ School of Management Engineering and Business, Hebei University of Engineering, Handan, China
}

Correspondence should be addressed to Kai Zhang; i_kaizhang@163.com

Received 25 June 2021; Accepted 6 August 2021; Published 20 August 2021

Academic Editor: Lei Xie

Copyright ( 2021 Ziqi Yin and Kai Zhang. This is an open access article distributed under the Creative Commons Attribution License, which permits unrestricted use, distribution, and reproduction in any medium, provided the original work is properly cited.

Forecasting the depth of groundwater in arid and semiarid areas is a great challenge because these areas are complex hydrogeological environments and the observational data are limited. To deal with this problem, the grey seasonal index model is proposed. The seasonal characteristics of time series were represented by indicators, and the grey model with fractional-order accumulation was employed to fit and forecast different periodic indicators and long-term trends, respectively. Then, the prediction results of the two were combined together to obtain the prediction results. To verify the model performance, the proposed model is applied to groundwater prediction in Yinchuan Plain. The results show that the fitting error of the proposed model is $2.08 \%$, while for comparison, the fitting error of the grey model of data grouping and Holt-Winters model is $3.94 \%$ and $5 \%$, respectively. In the same way, it is concluded that the fitting error of groundwater in Weining Plain by the proposed model is $2.26 \%$. On the whole, the groundwater depth in Ningxia Plain including Yinchuan Plain and Weining Plain will increase further.

\section{Introduction}

1.1. Motivation. Groundwater is closely related to the development of human civilization. More than 2 billion people worldwide drink groundwater directly. This number is rising as rivers are polluted and depleted. The overuse of groundwater destroys the natural water cycle and causes problems such as ground collapse. China's groundwater resources have been drastically reduced due to excessive exploitation of groundwater. At present, the accumulated deficit of groundwater deficit in North China is 180 billion cubic meters. In order to reduce the impact of groundwater depth decline, it is meaningful to achieve accurate groundwater prediction. Especially in dry areas, the reliance on groundwater is greater.

Ningxia Hui Autonomous Region (Ningxia) is located in Northwest China and is a typical dry area. Ningxia has the fewest water resources in China. The average annual runoff in Ningxia is $18.3 \mathrm{~mm}$ deep, only $1 / 15$ of the national average, with annual rainfall from $150 \mathrm{~mm}$ to $600 \mathrm{~mm}$. To solve the problem of farmland irrigation, the ancient people of Ningxia built water conservancy projects to divert the Yellow River. The water conservancy project is still in use today. In 2017, the ancient irrigation area in Ningxia was listed on the world irrigation engineering heritage list. Although irrigation projects have eased the drought on farmland, groundwater is still the main source of water resources in Ningxia. Moreover, with the continuous development of China's western development strategy, Ningxia's economy has developed rapidly, which has intensified the demand for water resources. According to Ningxia water resource bulletin, the groundwater consumption in Ningxia has been on the rise from 2014 to 2018. The content comes from Ningxia Hydrological Information Network.

In order to provide reference for groundwater management in Ningxia, the grey seasonal index model (GSIM $(1,1))$ was established to predict the groundwater depth of Ningxia Plain and compared with the results of HoltWinters model (Holt-Winters) and grey model of data grouping (DGGM $(1,1))$. 
This paper is organized as follows. The model is introduced in Section 2. Experimental results are given in Section 3. In Section 4, some conclusions and directions for future research are provided.

1.2. Literature Review. Under the background of water resource depletion, groundwater management has gradually developed into a discipline to be studied by scholars [1]. Accurate prediction of groundwater is important for groundwater management. There are many studies on prediction method [2]. The most direct way is through the surface vegetation to predict groundwater depth [3]. However, changes in groundwater are most affected by humans. Industrial water, agricultural water, and domestic water are considered as the main factors affecting groundwater [4]. Based on different influencing factors, stochastic time series and artificial neural network models are considered to have better effect in groundwater depth assessment [5]. Rivers are the main natural way to replenish groundwater and play a regulating role in the water cycle. A groundwater prediction model based river stage was built [6]. Based on groundwater dynamic data and related factors, the quantile regression method was used to predict groundwater depth [7]. Other factors affecting groundwater depth have also been investigated [8]. The development of science and technology provides convenience for the study of groundwater. The conceptual model, the Bayesian network, and artificial network models were applied to groundwater depth prediction [9]. Support vector machines have good performance in groundwater depth prediction [10]. The prediction effects of grey self-memory model, radial basis function network, and adaptive neuro fuzzy inference system models on groundwater were compared [11]. The mathematical models have their own characteristics, and different models are combined and applied to groundwater depth prediction [12]. Hybrid support vector machine regression and artificial neural network models were applied to groundwater depth prediction [13]. A hybrid model using entropy spectral analysis with the optimal input is proved to be effective in predicting groundwater depth [14]. The empirical mode decomposition (EMD) was combined with Elman neural network, and the coupling forecasting model was constructed to groundwater depth forecasting [15]. The EMD is also used in combination with phase space reconstruction, particle swarm optimization, and extreme learning machine [16].

Groundwater is a huge system. Due to the influence of geological conditions, hydrological environment, and other factors [17-20], the information about groundwater is ambiguous. Therefore, groundwater is a complex grey system. Moreover, the above model needs a lot of data to guarantee the accuracy of prediction [21]. Without a large number of training samples, the prediction accuracy of the model cannot reach the best [22]. The grey prediction model has high precision in small sample prediction [23, 24]. Grey models are widely used in air pollution, energy, electricity, biology, computer, water resources, and other fields [25-33]. The fractional-order grey prediction model improves the stability of the model from the perspective of fractional order $[34,35]$. On this basis, the seasonal index is introduced into the fractional-order grey prediction model, and the processing ability of the model to nonlinear data is improved. Thus, PSO-GSIM $(1,1)$ is used to predict the groundwater depth of Ningxia Plain.

\section{Methodology}

2.1. The Holt-Winters Model. The Holt-Winters model is a traditional statistical model and has been widely used since it was proposed by Winters in 1960. Scholars have conducted in-depth research on the model [36]. The Holt-Winters model is used to process data with seasonal characteristics. Time series data are divided into three parts, namely, residual data, trend data, and seasonal data. A prediction model is established based on the Holt-Winters model. There are two kinds of calculation methods for the HoltWinters model, namely, addition and multiplication. The multiplication Holt-Winters model works better with solving the seasonal time series prediction problem. Therefore, the multiplication Holt-Winters model is used for comparison in this paper. The establishment process of the model is as follows.

For the time series $X^{(0)}=\left\{x^{(0)}(1), x^{(0)}(2), \ldots, x^{(0)}(n)\right\}$, the multiplication Holt-Winters model is

$$
\begin{aligned}
& S_{t}=\alpha \frac{x^{(0)}(t)}{I_{t-L}}+(1-\alpha)\left(S_{t-1}+b_{t-1}\right), \quad 0<\alpha<1, \\
& b_{t}=\gamma\left(S_{t}-S_{t-1}\right)+(1-\gamma) b_{t-1}, \quad 0<\gamma<1, \\
& I_{t}=\beta \frac{x^{(0)}(t)}{S_{t}}(1-\beta) I_{t-L}, \quad 0<\beta<1,
\end{aligned}
$$

where $L$ is the seasonal length, such as four quarters, 12 months, and so on, $I_{t}$ is the seasonal index, and $b_{t}$ represents the tendency.

$$
\begin{aligned}
I_{t} & =\frac{\overline{x^{(0)}(L)}}{\overline{x^{(0)}(t)}} \\
S_{L+1} & =x^{(0)}(L+1), \\
b_{L+1} & =\frac{x^{(0)}(L+1)-x^{(0)}(1)+x^{(0)}(L+2)-x^{(0)}(2)+x^{(0)}(L+3)-x^{(0)}(3)}{3 L},
\end{aligned}
$$


where $\overline{x^{(0)}(L)}$ represents the average of same month in different years and $\overline{x^{(0)}(t)}$ is general average.

The Holt-Winters model has three parameters, and the application of the grey wolf optimization algorithm (GWO) improves the efficiency of multiparameter optimization. GWO is an optimization algorithm inspired by the hunting behavior of wolves. It is widely used for multiparameter model optimization. The behavior of a pack of wolves is represented digitally as follows:

$$
\begin{aligned}
D & =\left|C \cdot x_{p}(i)-x(i)\right|, \\
x(i+1) & =x_{p}(i)-A \cdot D,
\end{aligned}
$$

where $i$ represents the literation at this point, $A$ and $D$ are coefficient vectors, and $x_{p}$ represents the position vector of the prey, i.e. objective vector. $x(i+1)$ is the position of one of the wolves in the pack. Coefficient vectors $A$ and $D$ are obtained by the following formula:

$$
\begin{aligned}
& A=2 a \cdot r_{1}-a, \\
& C=2 \cdot r_{2},
\end{aligned}
$$

where $a$ decreases linearly from 2 to 0 along with iteration and $r_{1}$ and $r_{2}$ are randomly generated from $[0,1]$.

In GWO, there are three optimization parameters, $\alpha, \beta$, and $\delta$, corresponding to the three variables in the HoltWinters model, respectively, where alpha is the dominant player in GWO. Suppose that the three wolves knew where their prey was; therefore, the first three best hunting sites were preserved. Other searcher is forced to update their locations on this basis. The other search location update formula is as follows:

$$
\begin{gathered}
D_{\alpha}=\left|C_{1} \cdot x_{\alpha}(i)-x(i)\right|, \\
D_{\beta}=\left|C_{2} \cdot x_{\beta}(i)-x(i)\right|, \\
D_{\delta}=\left|C_{3} \cdot x_{\delta}(i)-x(i)\right|, \\
x_{1}(i+1)=x_{\alpha}(i)-A_{1} \cdot\left(D_{\alpha}\right), \\
x_{2}(i+1)=x_{\beta}(i)-A_{2} \cdot\left(D_{\beta}\right), \\
x_{3}(i+1)=x_{\delta}(i)-A_{3} \cdot\left(D_{\delta}\right), \\
x(i+1)=\frac{x_{1}(i+1)+x_{2}(i+1)+x_{3}(i+1)}{3},
\end{gathered}
$$

where $x(i)$ is the position of other searcher, $x_{\alpha}, x_{\beta}$, and $x_{\delta}$ are the search locations of alpha, beta, and delta, and $x(i+1)$ is where the other searchers are in the next iteration. To speed up search efficiency, the maximum number of iterations is set to 30 . The value range of parameters is $[0,1]$. Detailed description and source codes of GWO can be found in the literature [37].

2.2. Grey Model of Data Grouping Method. The traditional grey prediction model is not applicable to the data of seasonal fluctuation. For the grey model to process the seasonal data, DGGM $(1,1)$ model was proposed [38]. Two more steps are added to the traditional GM $(1,1)$ model. The calculation process of DGGM $(1,1)$ model is described below.

Step 1. The time series data are divided into twelve monthly groups and are given by

$$
\begin{array}{r}
x^{(0)}(\tau)=\left(x^{(0)}(\tau, 1), x^{(0)}(\tau, 2), \ldots, x^{(0)}(\tau, n)\right), \\
\tau=1,2, \ldots, 12 .
\end{array}
$$

Step 2. GM $(1,1)$ models are calculated based on grouped data separately. An accumulative series $x^{(1)}(\tau)$ is obtained by accumulative calculation:

$$
\begin{aligned}
x^{(1)}(\tau) & =\left\{x^{(1)}(\tau, 1), x^{(1)}(\tau, 2), \ldots, x^{(1)}(\tau, n)\right\} \\
& =\left\{x^{(1)}(\tau, 1), x^{(1)}(\tau, 1)+x^{(0)}(\tau, 2), \ldots, x^{(1)}(\tau, n-1)+x^{(0)}(\tau, n-1)\right\}, \quad \tau=1,2, \ldots, 12,
\end{aligned}
$$

where $x^{(1)}(\tau, k)=\sum_{i=1}^{k} x^{(0)}(\tau, i), k=1,2, \ldots, n ; \tau=1,2$, $\ldots, 12$, and the mean series is $z^{(1)}(\tau, k)=0.5 x^{(1)}(\tau, k)+$ $0.5 x^{(1)}(\tau, k-1) k=2,3, \ldots, n ; \tau=1,2, \ldots, 12$.
The first-order differential equation of a single variable is used as the prediction model, namely, GM $(1,1)$, and the grey differential equation is as follows: 


$$
\begin{aligned}
& x^{(0)}(\tau, k)+a z^{(1)}(\tau, k)=b, \\
k= & 2,3, \ldots, n ; \tau=1,2, \ldots, 12 .
\end{aligned}
$$

The corresponding whitening differential equation is $\left(\mathrm{d} x^{(1)}(\tau, t) / \mathrm{d} t\right)+a x^{(1)}(\tau, t)=b$, where $t, a$, and $b$ represent the time variable, grey development coefficient, and grey action quantity, respectively. Data matrix $B$ and data vector $Y$ are given by

$$
\begin{gathered}
B=\left[\begin{array}{cc}
-\frac{1}{2}\left[x^{(1)}(\tau, 1)+x^{(1)}(\tau, 2)\right] & 1 \\
-\frac{1}{2}\left[x^{(1)}(\tau, 2)+x^{(1)}(\tau, 3)\right] & 1 \\
\vdots & \vdots \\
-\frac{1}{2}\left[x^{(1)}(\tau, n-1)+x^{(1)}(\tau, n)\right] & 1
\end{array}\right], \\
Y=\left[\begin{array}{c}
\left.x^{(0)}(\tau, 2)\right] \\
x^{(0)}(\tau, 3) \\
\vdots \\
x^{(0)}(\tau, n)
\end{array}\right], \quad \tau=1,2, \ldots, 12 .
\end{gathered}
$$

$$
\hat{x}^{(0)}(\tau, t+1)=\left(\hat{x}^{(0)}(1,1), \hat{x}^{(0)}(2,1), \hat{x}^{(0)}(3,1), \hat{x}^{(0)}\right.
$$

$(4,1)$

2.3. The GSIM $(1,1)$ Model. The data varied substantially among different months, but the traditional grey prediction model is only suitable for time series with an exponential trend, and it is not capable of effectively predicting data with large fluctuations. Thus, in order to enhance the forecasting accuracy, the seasonal data are summarized into annual data in this study. The annual data are predicted by GM $(1,1)$ with fractional-order accumulation (FGM $(1,1))$ [24]. The prediction results were calculated by seasonal index and annual data.

The time series data on the depth of groundwater are $X^{(0)}=\left\{x^{(0)}(1), x^{(0)}(2), \ldots, x^{(0)}(n)\right\}$ with a cycle of $L$, for example, $L=12$, and the modeling process for the GSIM $(1,1)$ model is as follows.

Step 1. The yearly data are $Y^{(0)}=\left\{y^{(0)}(1), y^{(0)}(2)\right.$, $\left.\ldots, y^{(0)}(\phi)\right\}, \phi=[n / L]$, where $y^{(0)}(1)=\sum_{i=1}^{L} x^{(0)}(i)$, $y^{(0)}(2)=\sum_{i=L+1}^{2 L} x^{(0)}(i), \ldots, y^{(0)}(\phi)=\sum_{i=(\phi-1) L+1}^{n} x^{(0)}$ (i). $\varphi$ is the number of periods.

Step 2. By using $y^{(r)}(k)=\sum_{j=1}^{k} C_{k-j+r-1}^{k-j} y^{(0)}(j)$, the $r$-order accumulation sequence is

$$
Y^{(r)}=\left\{y^{(r)}(1), y^{(r)}(2), \ldots, y^{(r)}(\varphi),\right.
$$

The undetermined coefficient vector is $\left[\begin{array}{l}\hat{a} \\ \hat{b}\end{array}\right]=\left(B^{T} B\right)^{-1} B^{T} Y$, and the prediction equation is obtained:

$$
\begin{array}{r}
\hat{x}^{(1)}(\tau, t+1)=\left[x^{(0)}(\tau, 1)-\frac{b}{a}\right] e^{-a t}+\frac{b}{a}, \\
\tau=1,2, \ldots, 12 .
\end{array}
$$

Step 3. Reductive treatment is conducted. Through a subtraction calculation, the predicted values of original series $x^{(0)}$ are obtained:

$$
\begin{array}{r}
\hat{x}^{(0)}(\tau, t+1)=\hat{x}^{(1)}(\tau, t+1)-\hat{x}^{(1)}(\tau, t), \\
t=1,2, \ldots, n-1 ; \tau=1,2, \ldots, 12 .
\end{array}
$$

Furthermore, the twelve groups of monthly predictions are combined into a new time series: where $C_{r-1}^{0}=1, C_{k}^{k+1}=0, \quad C_{k-j+r-1}^{k-j}=((k-j+r-1)$ $(k-j+r-2) \ldots(r+1) r) /(k-j) ! . r$ is the fractional order, and the optimal value can be obtained through particle swarm optimization (PSO) algorithm.

Step 3. The differential equation with one variable of the $r$-order accumulation sequence $Y^{(r)}$ (i.e., the FGM $(1,1)$ model) can be defined as

$$
\frac{\mathrm{d} y^{(r)}}{\mathrm{d} t}+a y^{(r)}=b
$$

where $a$ and $b$ represent grey development coefficient and grey action quantity, respectively. The solution to equation (14) is

$$
y^{(r)}(t+1)=\left(y^{(0)}(1)-\frac{b}{a}\right) e^{-a t}+\frac{b}{a} .
$$

The parameters of the model are calculated by the least square method. The calculation process of $\hat{a}, b$ is shown in the following equation:

$$
\left[\begin{array}{l}
\hat{a} \\
\hat{b}
\end{array}\right]=\left(B^{\mathrm{T}} B\right)^{-1} B^{\mathrm{T}} Y
$$


where

$$
\begin{aligned}
& B=\left[\begin{array}{cc}
-0.5\left(y^{(r)}(1)+y^{(r)}(2)\right) & 1 \\
-0.5\left(y^{(r)}(2)+y^{(r)}(3)\right) & 1 \\
\vdots & \vdots \\
-0.5\left(y^{(r)}(\varphi-1)+y^{(r)}(\varphi)\right) & 1
\end{array}\right], \\
& Y=\left[\begin{array}{c}
y^{(r)}(2) \\
y^{(r)}(3) \\
\vdots \\
y^{(r)}(\varphi)
\end{array}\right] .
\end{aligned}
$$

Step 4. $\hat{a}$ and $\hat{b}$ are entered in the time response function:

$$
\hat{y}^{(r)}(k+1)=\left(y^{(0)}(1)-\frac{\hat{b}}{\hat{a}}\right) e^{-\hat{a} k}+\frac{\hat{b}}{\hat{a}},
$$

and $\hat{y}^{(r)}(k+1)$ is the fitting value at time $k+1$.

Step 5. For $\hat{Y}^{(r)}=\left\{\hat{y}^{(r)}(1), \hat{y}^{(r)}(2), \ldots, \hat{y}^{(r)}(\varphi)\right\}$, the restored sequence is

$\alpha^{(r)} \hat{Y}^{(r)}=\left\{\alpha^{(1)} \hat{y}^{(r)(1-r)}(1), \alpha^{(1)} \hat{y}^{(r)(1-r)}(2), \ldots, \alpha^{(1)} \hat{y}^{(r)(1-r)}(\varphi)\right\}$,

where $\alpha^{(1)} \hat{y}^{(r)(1-r)}(k)=\hat{y}^{(r)(1-r)}(k)-\hat{y}^{(r)(1-r)}(k-1)$. Then, the restored value is

$\hat{y}^{(0)}(1), \hat{y}^{(0)}(2), \ldots, \hat{y}^{(0)}(\varphi), \hat{y}^{(0)}(\varphi+1), \hat{y}^{(0)}(\varphi+2), \ldots$

Step 6. The seasonal index $\mathrm{d}(i, t)(i=1,2, \ldots, L, t=$ $1,2, \ldots, \phi)$ is calculated by using the average method:

$$
\mathrm{d}^{(0)}(i, t)=\left\{\mathrm{d}^{(0)}(1,1), \mathrm{d}^{(0)}(2,1), \ldots, \mathrm{d}^{(0)}(L, 1), \mathrm{d}^{(0)}(1,2), \ldots, \mathrm{d}^{(0)}(L, \varphi)\right\}
$$

where

$$
\begin{aligned}
\mathrm{d}^{(0)}(1,1) & =\frac{x^{(0)}(1,1)}{\sum_{i=1}^{L} x^{(0)}(i, 1)}, \mathrm{d}^{(0)}(2,1)=\frac{x^{(0)}(2,1)}{\sum_{i=1}^{L} x^{(0)}(i, 1)}, \ldots, \mathrm{d}^{(0)}(L, 1)=\frac{x^{(0)}(L, 1)}{\sum_{i=1}^{L} x^{(0)}(i, 1)}, \mathrm{d}^{(0)}(1,2) \\
& =\frac{x^{(0)}(1,2)}{\sum_{i=1}^{L} x^{(0)}(i, 2)}, \cdots, \mathrm{d}^{(0)}(L, \varphi)=\frac{x^{(0)}(L, \varphi)}{\sum_{i=1}^{L} x^{(0)}(i, \varphi)} .
\end{aligned}
$$

Step 7. According to the calculation process in Step 2, the accumulation series $\mathrm{d}^{(r)}(i, t)$ is obtained.

$\mathrm{d}^{(r)}(i, t)=\left\{\begin{array}{cccc}\mathrm{d}^{(r)}(1,1), & \mathrm{d}^{(r)}(1,2), & \ldots & \mathrm{d}^{(r)}(1, \varphi) ; \\ \mathrm{d}^{(r)}(2,1), & \mathrm{d}^{(r)}(2,2), & \ldots & \mathrm{d}^{(r)}(2, \varphi) ; \\ \vdots & & \\ \mathrm{d}^{(r)}(L, 1), & \mathrm{d}^{(r)}(L, 2), & \ldots & \mathrm{d}^{(r)}(L, \varphi) .\end{array}\right.$
The accumulation of the seasonal index is no longer carried out in accordance with the time series, but data of the same month are combined into a group for the accumulation. The purpose of this is to reduce the dimension of the data so as to make full use of the grey model to process the small data. At the same time, it is also preparation for the prediction of the next cycle.

The next calculation refers to Steps 3-5, and the forecasting value is obtained:

$$
\hat{\mathrm{d}}^{(0)}(1, \varphi+1), \hat{\mathrm{d}}^{(0)}(2, \varphi+1), \ldots, \hat{\mathrm{d}}^{(0)}(L, \varphi+1), \hat{\mathrm{d}}^{(0)}(1, \varphi+2), \hat{\mathrm{d}}^{(0)}(2, \varphi+2), \ldots
$$

Step 8. The fitting value of the groundwater depth is obtained.

$$
\begin{aligned}
& \text { For } \stackrel{\wedge}{X}^{(0)}=\left\{\hat{x}^{(0)}(1), \hat{x}^{(0)}(2), \ldots, \hat{x}^{(0)}(n), \hat{x}^{(0)}(n+1),\right. \\
& \ldots\}, \text { the solution formula is }
\end{aligned}
$$




$$
\begin{cases}\hat{x}^{(0)}(k)=\hat{y}^{(0)}(\omega+1) \frac{\hat{\mathrm{d}}^{(0)}(v, \omega+1)}{L}, & \bmod (k, L) \neq 0 \\ \hat{x}^{(0)}(k)=\hat{y}^{(0)}(\omega) \frac{\hat{\mathrm{d}}^{(0)}(L, \omega)}{L}, & \bmod (k, L)=0\end{cases}
$$

where $\omega$ and $v$ are the quotient and remainder of $k / L$, respectively.

Step 9. The mean absolute percentage error (MAPE) is used to test model accuracy, and the calculation process is as follows:

$$
\text { MAPE }=\frac{1}{n} \sum_{k=1}^{n}\left|\frac{\hat{x}^{(0)}(k)-x^{(0)}(k)}{x^{(0)}(k)}\right| \times 100 \%
$$

In the paper, PSO is applied to obtain the optimal value of the parameter $r$ of the GSIM $(1,1)$ model. PSO has been widely used since it was proposed [39]. Compared with other optimization algorithms, PSO is simpler and easier to implement without too much parameter adjustment. To increase the search effect, the position and velocity of the particle need to be constantly updated. The updating process depends on the values Pbest and gbest, where Pbest represents the current optimal solution and gbest represents the current optimal solution for any particle. The updating process is shown in the following equations:

$$
\begin{aligned}
v(i+1)= & \omega v(i)+c_{1} * \text { rand } *(\operatorname{Pbest}(i)-x(i)) \\
& +c_{2} * \text { rand } *(\operatorname{gbest}(i)-x(i)), \\
x(i+1)= & x(i)+v(i+1),
\end{aligned}
$$

where $v(i)$ and $x(i)$ represent the velocity and position of the particle at $i$, respectively, $\omega$ represents the inertia weight value which is generally set as 0.8 [40], and rand is a random function; the optimal value of $r$ of most experiments is generally within the range of $[0,1]$; to ensure the accuracy of the experiment, the value range of $\operatorname{GSIM}(1,1)$ parameter $r$ is expanded to $[0,2.5]$; therefore, rand represents the random number generated from $[0,2.5] . c_{1}$ and $c_{2}$ are acceleration constants with equal values and set to 2 [41]. Suppose the number of individuals in the initial population is $N=50$, the maximum number of iterations is 200 . More detailed description of PSO can be found in literature [42].

\section{Experimental Results}

3.1. Study Area and Data. Ningxia spans $35^{\circ} 14^{\prime}-39^{\circ} 14^{\prime} \mathrm{N}$ and $104^{\circ} 17^{\prime}-109^{\circ} 39^{\prime} \mathrm{E}$ and includes five cities. The climatic types of Ningxia are temperate, continental, arid, and semiarid. Ningxia Plain is considered as the largest plain in Ningxia. The study region has an area of $66.400 \mathrm{~km}^{2}$ and is bordered by the Helan Mountains and Liupan Mountains to the northwestward and south, respectively. Ningxia is 1100-1200 meters above sea level and the terrain slopes from southwest to northeast. The Yellow River enters from the southwest of Ningxia and leaves from the northeast. Due to the different physiognomy types in northcentral Ningxia and south Ningxia, Ningxia Plain can be divided into Yinchuan Plain and Weining Plain (Figure 1). The recoverable groundwater accounts for $88 \%$ of the total groundwater in Ningxia. Ningxia Plain is surrounded by mountains, water flows to the plain, and the water table changes slowly and steadily, creating a predictable gradient of water [43].

The data are from the official website of the Ministry of Water Resources of the People's Republic of China (https:// www.mwr.gov.cn/sj/tjgb/dxsdtyb/). There are a total of 47 groundwater monitoring wells in Ningxia Plain. Yinchuan Plain contains three cities and 28 groundwater monitoring wells. Weining Plain contains one city and 11 groundwater monitoring wells (by the end of the 2019). The groundwater depth data for Yinchuan Plain and Weining Plain are the average result of multiple groundwater monitoring wells.

\subsection{Forecasting the Groundwater Depth of Yinchuan Plain.} The groundwater depth of Yinchuan Plain is predicted in this section. The depth of groundwater is one of the contents of hydrology. Groundwater is subject to changes in rainfall or in rivers. As we all know, changes in rainfall and rivers are cyclical and seasonal. In recent years, the seasonal variation of groundwater depth is more obvious under the influence of human production activities. For example, the original groundwater depth data of Yinchuan Plain are summarized in Figure 2. Figure 2 shows that the groundwater depth changes with time, and groundwater depth fluctuations are similar at the same time in different years. Therefore, the seasonal model is used to study the variation of groundwater depth.

\subsubsection{The Calculation Results of the Holt-Winters Model.} The groundwater depth data of Yinchuan Plain from 2014 to 2018 were fitted to three models. The models do not fit the data of the first period, so the data of the first period are omitted. The fitting results of the Holt-Winters model are listed in Table 1. The calculation process is completed by MATLAB 2016b. The GWO was used to find optimal parameters $\alpha, \beta$, and $\gamma$, and the optimization result of parameters is $[0.51639,0.05160,0.00752]$. The fitting error (MAPE) of the Holt-Winters model is $5.00 \%$. It can be seen that the fitting accuracy of the traditional model is lower than $10 \%$, and the fitting results are accepted. As the first cycle, the data of 2014 were omitted.

\subsubsection{The Calculation Results of the DGGM $(1,1)$ Model.} The DGGM $(1,1)$ model was applied to the data calculation on a monthly basis. The data grouping method is adopted to improve the fitting accuracy of the traditional GM $(1,1)$ model, and the model can be applied to process seasonal fluctuation data. The fitting results of DGGM $(1,1)$ model are listed in Table 2. MAPE is $3.94 \%$. Compared with the Holt-Winters model, the new model has higher fitting accuracy. Moreover, there are no fitting results for the first period of the two models, increasing contrast between models. 


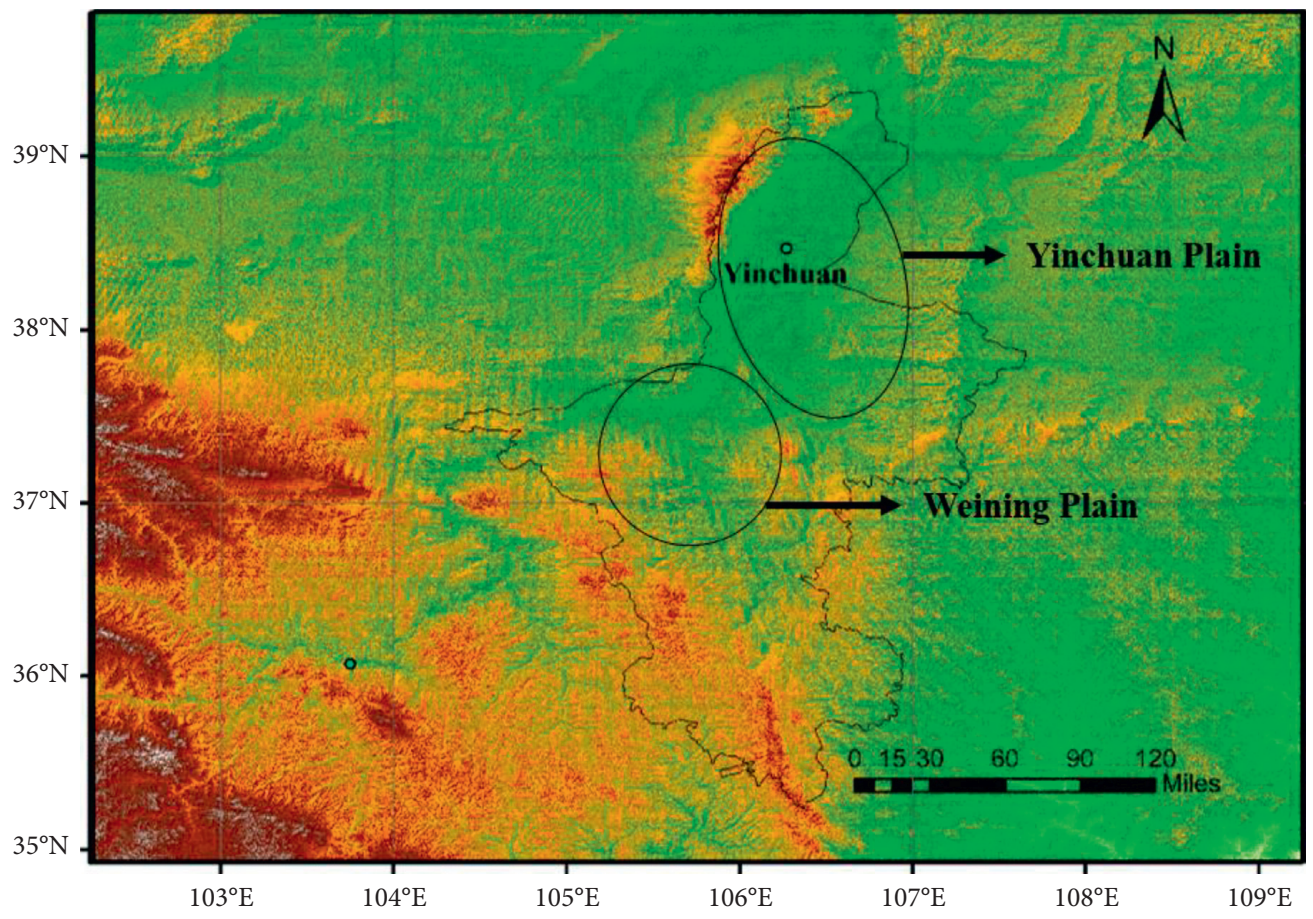

Figure 1: The general area of Ningxia Plain.

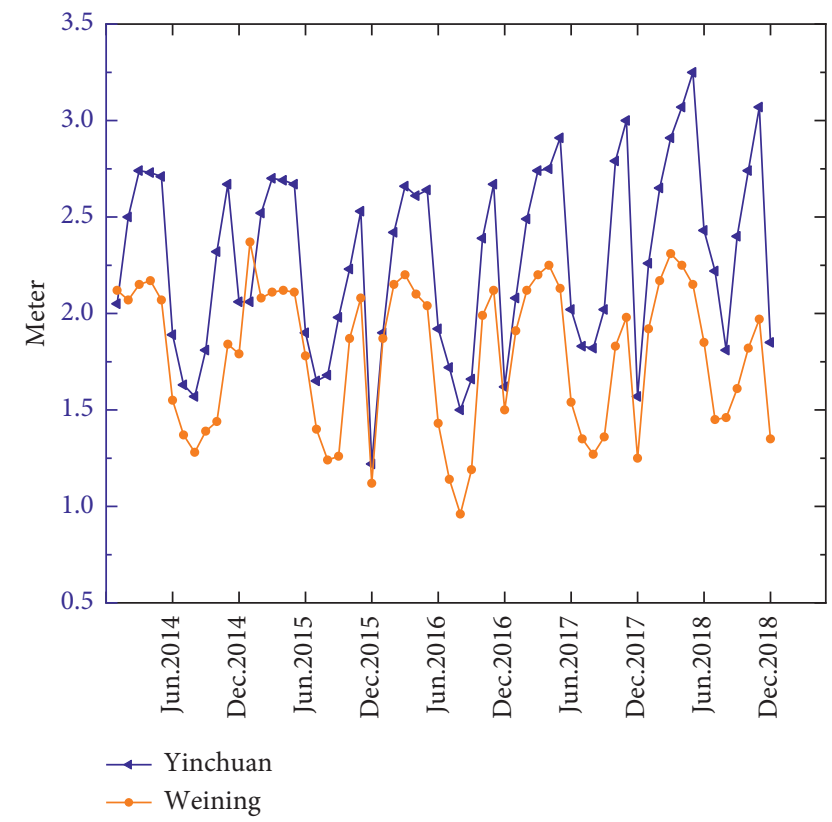

Figure 2: The groundwater depth of Yinchuan Plain and Weining Plain.

The parameters generated by the DGGM $(1,1)$ model in the fitting process are shown in Table 3 . The time response function of each set of parameters can be obtained from equation (16).

3.2.3. The Calculation Results of the GSIM $(1,1)$ Model. The seasonal index in this paper refers to the index calculated on a monthly basis. The seasonal index reflects the
TABLE 1: The fitting value of Yinchuan Plain groundwater depth by Holt-Winters model (unit: m).

\begin{tabular}{lllll}
\hline \multirow{2}{*}{ Month } & \multicolumn{5}{c}{ Year } \\
& 2015 & 2016 & 2017 & 2018 \\
\hline 1 & 2.06 & 1.69 & 2.00 & 2.09 \\
2 & 2.27 & 2.19 & 2.48 & 2.65 \\
3 & 2.63 & 2.53 & 2.71 & 2.89 \\
4 & 2.69 & 2.62 & 2.75 & 2.92 \\
5 & 2.75 & 2.68 & 2.81 & 3.07 \\
6 & 1.94 & 1.90 & 2.05 & 2.27 \\
7 & 1.71 & 1.70 & 1.81 & 2.10 \\
8 & 1.55 & 1.59 & 1.69 & 2.01 \\
9 & 1.91 & 1.81 & 2.07 & 2.23 \\
10 & 2.46 & 2.18 & 2.58 & 2.94 \\
11 & 2.61 & 2.57 & 3.02 & 3.16 \\
12 & 1.53 & 1.56 & 1.79 & 1.85 \\
MAPE & & & 5.00 & \\
\hline
\end{tabular}

fluctuation of the data in the time series. Most models do not deal well with fluctuating data. For the purpose of stabilizing the sequence, scholars used seasonal factors to eliminate seasonal changes in time series. This method improves the ability of the model to process the fluctuation data. However, the time series that eliminate seasonal effects may lose a lot of information. Its accuracy is also difficult to improve. To reduce information loss in the sequence, the seasonal index was extracted and studied separately. The results of yearly data are listed in Table 4 . The MAPE is $0.38 \%$, and the fitting accuracy is high.

Then, the seasonal index of monthly data is fitted. During the fitting process, the GSIM $(1,1)$ model is used sometimes, so the multiple sets of parameters are generated. Each set of 
TAвLE 2: The fitting value of Yinchuan Plain groundwater depth by DGGM $(1,1)$ model (unit: m).

\begin{tabular}{|c|c|c|c|c|}
\hline \multirow{2}{*}{ Month } & \multicolumn{4}{|c|}{ Year } \\
\hline & 2015 & 2016 & 2017 & 2018 \\
\hline 1 & 2.03 & 1.97 & 2.11 & 2.18 \\
\hline 2 & 2.38 & 2.51 & 2.45 & 2.62 \\
\hline 3 & 2.56 & 2.74 & 2.79 & 2.91 \\
\hline 4 & 2.64 & 2.67 & 2.84 & 3.09 \\
\hline 5 & 2.56 & 2.78 & 3.07 & 3.24 \\
\hline 6 & 1.83 & 2.08 & 2.14 & 2.28 \\
\hline 7 & 1.64 & 1.67 & 1.89 & 2.07 \\
\hline 8 & 1.61 & 1.74 & 1.67 & 1.78 \\
\hline 9 & 1.82 & 1.93 & 2.09 & 2.26 \\
\hline 10 & 2.33 & 2.41 & 2.62 & 2.77 \\
\hline 11 & 2.45 & 2.67 & 2.91 & 3.05 \\
\hline 12 & 1.31 & 1.45 & 1.63 & 1.8 \\
\hline MAPE & \multicolumn{4}{|c|}{3.94} \\
\hline
\end{tabular}

TABle 3: The parameters of the DGGM $(1,1)$ model fitting process.

\begin{tabular}{lcc}
\hline Modeling the serial number & Grey development coefficient $\widehat{a}$ & Grey action quantity $\widehat{b}$ \\
\hline 1 & -0.039 & 1.838 \\
2 & -0.019 & 2.381 \\
3 & -0.026 & 2.539 \\
4 & -0.047 & 2.395 \\
5 & -0.072 & 2.279 \\
6 & -0.085 & 1.574 \\
7 & -0.101 & 1.337 \\
8 & -0.043 & 1.495 \\
9 & -0.086 & 1.53 \\
10 & -0.075 & 2.003 \\
11 & -0.069 & 2.263 \\
12 & -0.116 & 0.992 \\
\hline
\end{tabular}

TABLE 4: The annual fitting value of Yinchuan Plain groundwater depth (unit: $\mathrm{m}$ ).

\begin{tabular}{lcc}
\hline Year & Actual value & Fitting value \\
\hline 2014 & 26.68 & 26.68 \\
2015 & 25.83 & 25.81 \\
2016 & 25.71 & 25.70 \\
2017 & 28.02 & 27.70 \\
2018 & 30.66 & 30.74 \\
MAPE & & 0.38 \\
\hline
\end{tabular}

parameters in Table 5 can be substituted into equation (20) to obtain the corresponding time response function.

The fitting values of the seasonal indices are listed in Table 6. The average MAPE from 2015 to 2018 is $2.01 \%$. Groundwater is consumed more in February, March, April, May, October, and November. Affected by dry and wet seasons, the groundwater depth varies seasonally throughout the year. The GSIM $(1,1)$ model can not only predict accurately but also quantify the periodic variation of data within the time span. With the allowable error, it is beneficial to understand the variation law of groundwater depth.

The groundwater depth fitting results from 2014 to 2018 are shown in Table 7. The MAPE of fitting results is $2.08 \%$. By calculating the seasonal index and annual fitting value of
TABLE 5: The parameters of the GSIM $(1,1)$ model fitting process in Yinchuan Plain.

\begin{tabular}{lccc}
\hline $\begin{array}{l}\text { Modeling the serial } \\
\text { number }\end{array}$ & $\begin{array}{c}\text { The optimal order } \\
\text { number } r\end{array}$ & $\hat{a}$ & $\hat{b}$ \\
\hline 1 & 1.21 & -0.05 & 1.08 \\
2 & 0.99 & 0.05 & 1.24 \\
3 & 0.15 & 0.89 & 1.40 \\
4 & 1.13 & -0.03 & 1.35 \\
5 & 1.10 & -0.04 & 1.28 \\
6 & 1.10 & -0.05 & 0.90 \\
7 & 1.11 & -0.07 & 0.77 \\
8 & 1.25 & -0.07 & 0.87 \\
9 & 1.58 & -0.19 & 1.11 \\
10 & 0.54 & 0.18 & 0.83 \\
11 & 0.57 & 0.17 & 0.95 \\
12 & 0.98 & -0.05 & 0.55 \\
\hline
\end{tabular}

groundwater depth, the monthly fitting value is obtained. As the trend parameter, the annual fitting value controls the change direction of groundwater. The seasonal index breaks down the units of time from years into months.

In the calculation process of the above three models, the GSIM $(1,1)$ model extracts the most information, and the seasonal variation of the data is shown in the prediction 
TABLe 6: Seasonal index fitting value of groundwater depth in Yinchuan Plain.

\begin{tabular}{|c|c|c|c|c|c|c|c|c|c|}
\hline \multirow{2}{*}{ Month } & \multirow{2}{*}{$\begin{array}{c}2014 \\
\text { Actual value }\end{array}$} & \multicolumn{2}{|c|}{2015} & \multicolumn{2}{|c|}{2016} & \multicolumn{2}{|c|}{2017} & \multicolumn{2}{|c|}{2018} \\
\hline & & Actual value & Fitting value & Actual value & Fitting value & Actual value & Fitting value & Actual value & Fitting value \\
\hline 1 & 0.92 & 0.96 & 0.96 & 0.89 & 0.89 & 0.89 & 0.88 & 0.88 & 0.89 \\
\hline 2 & 1.12 & 1.17 & 1.17 & 1.13 & 1.12 & 1.07 & 1.08 & 1.04 & 1.03 \\
\hline 3 & 1.23 & 1.25 & 1.25 & 1.24 & 1.22 & 1.17 & 1.18 & 1.14 & 1.14 \\
\hline 4 & 1.23 & 1.25 & 1.25 & 1.22 & 1.21 & 1.18 & 1.20 & 1.20 & 1.19 \\
\hline 5 & 1.22 & 1.24 & 1.24 & 1.23 & 1.23 & 1.25 & 1.25 & 1.27 & 1.27 \\
\hline 6 & 0.85 & 0.88 & 0.88 & 0.90 & 0.88 & 0.87 & 0.90 & 0.95 & 0.93 \\
\hline 7 & 0.73 & 0.77 & 0.77 & 0.80 & 0.78 & 0.78 & 0.81 & 0.87 & 0.85 \\
\hline 8 & 0.71 & 0.78 & 0.78 & 0.70 & 0.73 & 0.78 & 0.72 & 0.71 & 0.74 \\
\hline 9 & 0.81 & 0.92 & 0.92 & 0.77 & 0.77 & 0.87 & 0.84 & 0.94 & 0.96 \\
\hline 10 & 1.04 & 1.04 & 1.07 & 1.12 & 1.11 & 1.19 & 1.12 & 1.07 & 1.10 \\
\hline 11 & 1.20 & 1.18 & 1.20 & 1.25 & 1.24 & 1.28 & 1.24 & 1.20 & 1.22 \\
\hline 12 & 0.93 & 0.57 & 0.63 & 0.76 & 0.66 & 0.67 & 0.70 & 0.72 & 0.73 \\
\hline MAPE & & & & & 2.01 & & & & \\
\hline
\end{tabular}

TABLE 7: The fitting value of Yinchuan Plain groundwater depth by GSIM $(1,1)$ model (unit: $\mathrm{m})$.

\begin{tabular}{lccccc}
\hline Month & & \multicolumn{5}{c}{ Year } \\
& 2014 & 2015 & 2016 & 2017 & 2018 \\
\hline 1 & 2.05 & 2.06 & 1.91 & 2.03 & 2.27 \\
2 & 2.49 & 2.52 & 2.41 & 2.49 & 2.65 \\
3 & 2.73 & 2.68 & 2.61 & 2.72 & 2.92 \\
4 & 2.73 & 2.69 & 2.60 & 2.76 & 3.05 \\
5 & 2.71 & 2.67 & 2.64 & 2.88 & 3.26 \\
6 & 1.89 & 1.89 & 1.89 & 2.08 & 2.39 \\
7 & 1.62 & 1.66 & 1.68 & 1.88 & 2.18 \\
8 & 1.58 & 1.68 & 1.55 & 1.67 & 1.90 \\
9 & 1.80 & 1.98 & 1.65 & 1.93 & 2.45 \\
10 & 2.31 & 2.29 & 2.38 & 2.58 & 2.83 \\
11 & 2.67 & 2.58 & 2.65 & 2.86 & 3.12 \\
12 & 2.07 & 1.35 & 1.42 & 1.61 & 1.88 \\
MAPE & & & 2.08 & & \\
\hline
\end{tabular}

process. The prediction accuracy of the GSIM $(1,1)$ model is also higher than that of the GWO-Holt-Winters model and the DGGM $(1,1)$ model. The MAPE of the GSIM $(1,1)$ model is $2.08 \%$, the MAPE of the Holt-Winters and the DGGM $(1,1)$ models is $5 \%$ and $3.94 \%$, respectively (Table 8 ). This shows that the GSIM $(1,1)$ model is more practical for seasonal fluctuation data.

The fitting process of the three models is visualized in Figure 3. The consumption of groundwater in Ningxia keeps increasing, and seasonal fluctuation trend is obvious. The fitting line of the GSIM $(1,1)$ model is close to the original data line. The three models did not fit the data of the first period. It can be seen that the original calculation of the three models has the same characteristics, and the three models are comparable.

3.2.4. Prediction of Groundwater Depth in Yinchuan Plain. In the fitting results of groundwater depth in Yinchuan Plain, the GSIM $(1,1)$ model has the smallest error. Therefore, the GSIM $(1,1)$ model is selected to predict the groundwater depth of Yinchuan Plain from 2019 to 2020 (Table 9). From the prediction results, groundwater
TABLE 8: The fitting error comparison of three models.

\begin{tabular}{lc}
\hline Model & MAPE \\
\hline Holt-Winters & 5 \\
DGGM $(1,1)$ & 3.94 \\
GSIM $(1,1)$ & 2.08 \\
\hline
\end{tabular}

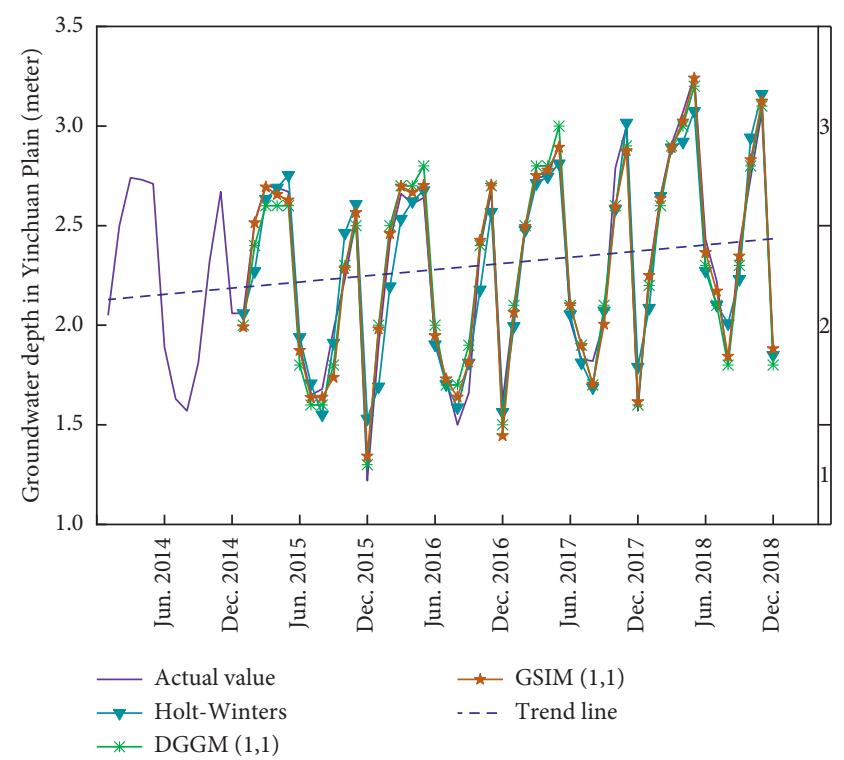

Figure 3: The fitting curve of groundwater depth in Yinchuan Plain.

Table 9: Prediction of groundwater depth in Yinchuan Plain (unit: $\mathrm{m}$ ).

\begin{tabular}{lccccc}
\hline \multirow{2}{*}{ Month } & \multicolumn{2}{c}{ Year } & Month & \multicolumn{2}{c}{ Year } \\
& 2019 & 2020 & & 2019 & 2020 \\
\hline 1 & 2.60 & 3.04 & 7 & 2.60 & 3.12 \\
2 & 2.86 & 3.12 & 8 & 2.23 & 2.65 \\
3 & 3.19 & 3.53 & 9 & 3.24 & 4.37 \\
4 & 3.44 & 3.92 & 10 & 3.10 & 3.40 \\
5 & 3.76 & 4.40 & 11 & 3.42 & 3.74 \\
6 & 2.79 & 3.30 & 12 & 2.22 & 2.65 \\
\hline
\end{tabular}


consumption in Yinchuan Plain will increase further. The seasonal law of groundwater depth remains unchanged. Ningxia is inland in Northwest China. The inland areas are short of water. Yinchuan Plain is the most economically dynamic area in Ningxia. Continued economic growth has increased water consumption. In addition, the tradition farmland irrigation model consumes most of the water resources. Strengthening water resources management is crucial to the economic development and agricultural production of Yinchuan Plain.

The GSIM $(1,1)$ model is the best among three models in Yinchuan Plain. This model can also predict the groundwater depth of Weining Plain. These results in Weining Plain and Yinchuan Plain help us to know the groundwater situation in the whole Ningxia Plain. The prediction of groundwater depth in Weining Plain is described in the next section.

\subsection{Forecasting the Groundwater Depth of Weining Plain.} Yinchuan, the capital of Ningxia, is located on the Yinchuan Plain. Zhongwei city is located on the Weining Plain. The Yellow River enters Ningxia from Zhongwei city. From a geographical point of view, Weining Plain has more water resources than Yinchuan Plain. The Yellow River flows from Weining Plain to Yinchuan Plain. Weining Plain needs to coordinate the local water resources to support the water shortage of Yinchuan Plain. Therefore, the accurate prediction of groundwater in Weining Plain is conductive not only to the regulation of local water resources but also to the diversion of water to Yinchuan Plain.

The annual groundwater depth in Weining Plain changes slowly. The annual data of groundwater depth were fitted by the FGM $(1,1)$ model. The fitting results are listed in Table 10 , and the MAPE of fitting value is $0.10 \%$ and has high precision. Since 2016, the groundwater depth of Weining Plain has gradually increased. It shows that water consumption in Weining Plain has increased in recent years.

The variation law of groundwater consumption in Weining Plain is known by seasonal index. The parameters generated by the GSIM $(1,1)$ model during the fitting process are listed in Table 11. The fitting results of the seasonal index are listed in Table 12, and the MAPE of fitting value is $2.23 \%$. The groundwater consumption in Weining Plain is large from January to May and November to December. The seasonal index in December showed a downward trend. It indicates that the groundwater consumption in December was reduced. But the seasonal index in October was above 1 several times. Changes in groundwater consumption are also dynamic, and it is important to predict the next year based on the index of same season.

The MAPE of the GSIM $(1,1)$ model for the groundwater depth of Weining Plain is marked in Figure 4. The error is obtained by averaging the monthly data fitting error of a year. The original value of groundwater depth in Weining Plain is represented by the line in Figure 4 . The seasonal variation of groundwater depth is obvious. The largest error occurred in 2016, which is $5.6 \%$. The overall level of error is low.
TABLE 10: Annual fitting value of groundwater depth in Weining Plain (unit: $\mathrm{m}$ ).

\begin{tabular}{lcc}
\hline Year & Actual value & Fitting value \\
\hline 2014 & 21.24 & 21.24 \\
2015 & 21.54 & 21.52 \\
2016 & 20.69 & 20.66 \\
2017 & 21.19 & 21.18 \\
2018 & 22.31 & 22.28 \\
MAPE & & 0.10 \\
\hline
\end{tabular}

TABLE 11: The parameters of the GSIM $(1,1)$ model fitting process in Weining Plain.

\begin{tabular}{lccc}
\hline $\begin{array}{l}\text { Modeling the serial } \\
\text { number }\end{array}$ & $\begin{array}{c}\text { The optimal order } \\
\text { number } r\end{array}$ & $\widehat{a}$ & $\hat{b}$ \\
\hline 1 & 1.39 & -0.08 & 1.62 \\
2 & 0.52 & 0.21 & 0.94 \\
3 & 0.55 & 0.17 & 0.91 \\
4 & 0.52 & 0.17 & 0.86 \\
5 & 0.14 & 0.50 & 0.81 \\
6 & 1.59 & -0.18 & 1.22 \\
7 & 1.56 & -0.17 & 0.97 \\
8 & 1.77 & -0.24 & 0.92 \\
9 & 1.44 & -0.18 & 0.81 \\
10 & 0.42 & 0.44 & 1.08 \\
11 & 0.47 & 0.37 & 1.13 \\
12 & 0.88 & 0.01 & 0.60 \\
\hline
\end{tabular}

TABle 12: Seasonal index fitting value of Weining Plain groundwater depth.

\begin{tabular}{lccccc}
\hline Month & & \multicolumn{5}{c}{ Year } \\
& 2014 & 2015 & 2016 & 2017 & 2018 \\
\hline 1 & 1.20 & 1.32 & 1.10 & 1.05 & 1.05 \\
2 & 1.17 & 1.18 & 1.21 & 1.20 & 1.17 \\
3 & 1.21 & 1.20 & 1.24 & 1.26 & 1.24 \\
4 & 1.23 & 1.19 & 1.22 & 1.23 & 1.22 \\
5 & 1.17 & 1.18 & 1.19 & 1.18 & 1.17 \\
6 & 0.88 & 0.99 & 0.82 & 0.87 & 0.99 \\
7 & 0.77 & 0.78 & 0.67 & 0.72 & 0.81 \\
8 & 0.72 & 0.69 & 0.56 & 0.67 & 0.82 \\
9 & 0.79 & 0.70 & 0.69 & 0.76 & 0.87 \\
10 & 0.81 & 1.06 & 1.09 & 1.05 & 0.99 \\
11 & 1.04 & 1.17 & 1.18 & 1.13 & 1.06 \\
12 & 1.01 & 0.71 & 0.72 & 0.74 & 0.75 \\
MAPE & & & 2.23 & & \\
\hline
\end{tabular}

From the fitting value of the year and the seasonal index, the fitting value of the month from 2014 to 2018 is calculated (Table 13). The prediction section in Table 13 is visualized in Figure 5. It can be seen that the predicted value has a small increase compared with the original data. The groundwater depth in 2019 to 2020 will still fluctuate seasonally. The groundwater consumption in the first half is more than the second half. Government departments should focus on water management in the first half of the year. 


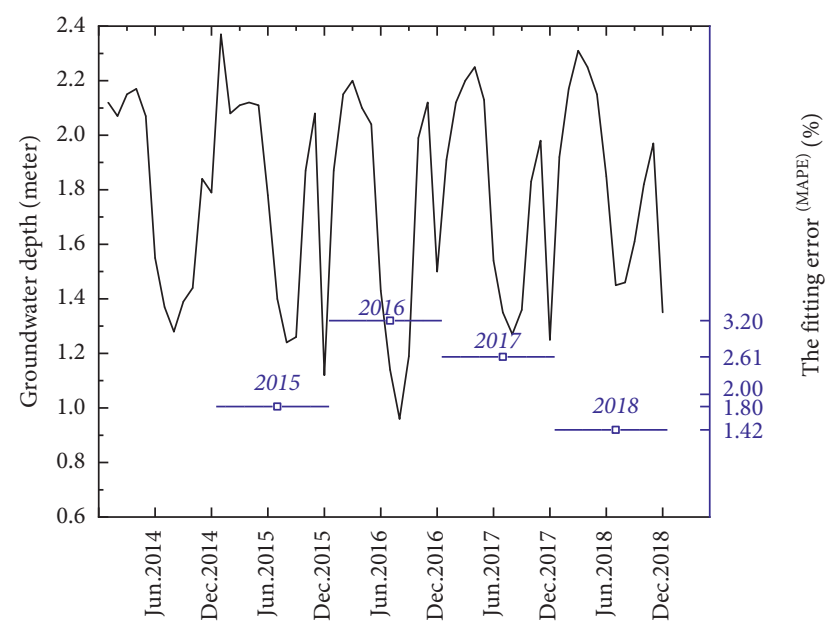

Figure 4: Annual fitting error value by the GSIM $(1,1)$ model.

TABLE 13: The fitting value and prediction value of groundwater depth in Weining Plain (unit: $\mathrm{m}$ ).

\begin{tabular}{lccccccc}
\hline \multirow{3}{*}{ Month } & \multicolumn{5}{c}{ Fitting value } & \multicolumn{3}{c}{$\begin{array}{c}\text { Prediction } \\
\text { value }\end{array}$} \\
& 2014 & 2015 & 2016 & 2017 & 2018 & 2019 & 2020 \\
\hline 1 & 2.12 & 2.37 & 1.89 & 1.85 & 1.95 & 2.14 & 2.39 \\
2 & 2.07 & 2.11 & 2.08 & 2.13 & 2.18 & 2.24 & 2.30 \\
3 & 2.14 & 2.15 & 2.14 & 2.22 & 2.31 & 2.41 & 2.51 \\
4 & 2.18 & 2.13 & 2.11 & 2.18 & 2.27 & 2.37 & 2.47 \\
5 & 2.07 & 2.11 & 2.04 & 2.09 & 2.17 & 2.27 & 2.40 \\
6 & 1.56 & 1.78 & 1.40 & 1.54 & 1.84 & 2.28 & 2.89 \\
7 & 1.36 & 1.40 & 1.15 & 1.26 & 1.50 & 1.84 & 2.31 \\
8 & 1.27 & 1.24 & 0.97 & 1.17 & 1.53 & 2.04 & 2.78 \\
9 & 1.40 & 1.25 & 1.19 & 1.34 & 1.61 & 2.00 & 2.52 \\
10 & 1.43 & 1.89 & 1.88 & 1.85 & 1.83 & 1.82 & 1.83 \\
11 & 1.84 & 2.10 & 2.03 & 2.00 & 1.97 & 1.96 & 1.95 \\
12 & 1.79 & 1.27 & 1.25 & 1.31 & 1.40 & 1.51 & 1.65 \\
MAPE & & & & 2.26 & & & \\
\hline
\end{tabular}

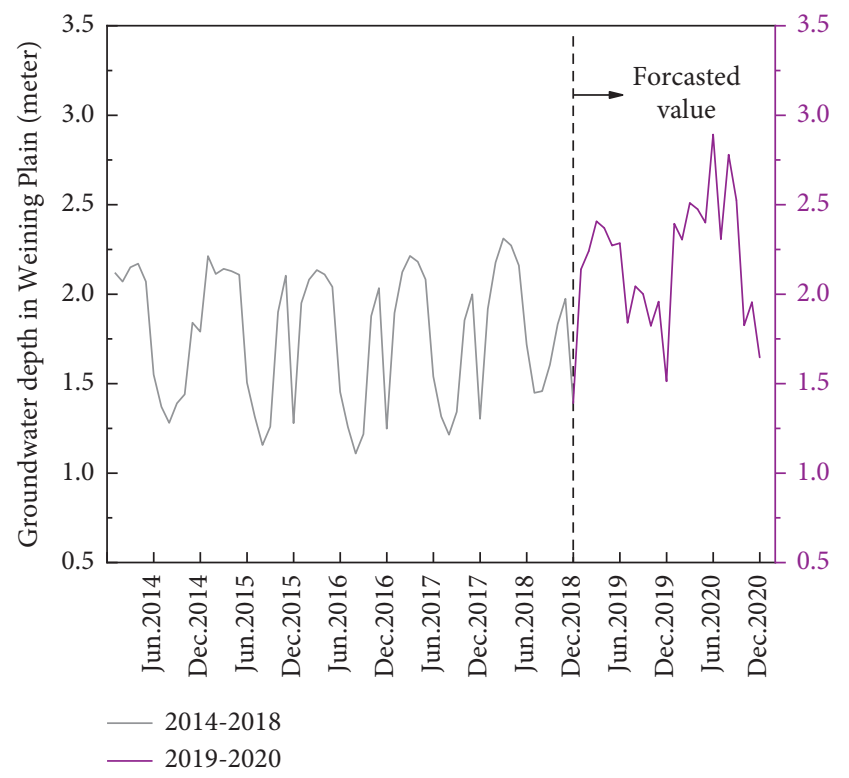

Figure 5: The results of groundwater depth fitting and prediction in Weining Plain.
The groundwater depth of Yinchuan Plain and Weining Plain is calculated and analyzed. It shows that the groundwater depth in Ningxia Plain is increasing year by year. Maintaining groundwater stability is important in dry areas. Water resources are coordinated between the two plains by the Yellow River. Weining Plain in the upper reaches of the Yellow River can allocate more water resources to Yinchuan Plain. The concept of coordinated development is also applied to water resources management.

\section{Conclusions}

In this paper, the GSIM $(1,1)$ model, the Holt-Winters model, and the DGGM $(1,1)$ model were established and applied to groundwater depth prediction in Ningxia Plain. By comparison, the GSIM $(1,1)$ model is proved to be an effective one, and its prediction accuracy is higher than that of other models. The following conclusions were obtained.

The GSIM $(1,1)$ model is more suitable for dealing with seasonal fluctuation data. In the process of calculation, the seasonal variation of data is presented. The $\operatorname{GSIM}(1,1)$ model provides a new choice for studying the data with the characteristics of seasonal variation.

GSIM $(1,1)$ retains the characteristics of the traditional grey model for processing small sample data and has high prediction accuracy. Seasonal variations in the data were quantified and used for the first time to predict research. Data for the same month for different years are used as the object of the GSIM $(1,1)$ model, and the processing method of traditional series data is changed.

In the paper, the GSIM $(1,1)$ model parameter $r$ is reserved for two decimal places. During the experiment, we found that the more the bits reserved for parameter $r$, the higher the accuracy of the model. Scholars can adjust parameter $r$ according to the experimental needs to meet higher accuracy requirements.

\section{Data Availability}

The data used to support the findings of this study are included within the article.

\section{Conflicts of Interest}

The authors declare that they have no conflicts of interest.

\section{Acknowledgments}

This study was supported by the National Natural Science Foundation of China (U20A20316).

\section{References}

[1] Y. Wada, L. P. Van Beek, C. M. Van Kempen, J. W. Reckman, S. Vasak, and M. F. Bierkens, "Global depletion of groundwater resources," Geophysical Research Letters, vol. 37, no. 20, 2010.

[2] S. Eslamian, Forecasting, Encyclopedia of Energy, Salem Press, Pasadena, CA, USA, 2012. 
[3] X.-D. Yang, Y.-D. Qie, D.-X. Teng et al., "Prediction of groundwater depth in an arid region based on maximum tree height," Journal of Hydrology, vol. 574, pp. 46-52, 2019.

[4] Y. Zhou, W. Xiao, J. Wang et al., "Evaluating spatiotemporal variation of groundwater depth/level in Beijing plain, a groundwater-fed area from 2001 to 2010," Advances in Meteorology, Article ID 8714209, 2016.

[5] B. Agoubi and A. Kharroubi, "Groundwater depth monitoring and short-term prediction: applied to El Hamma aquifer system, southeastern Tunisia," Arabian Journal of Geosciences, vol. 12, no. 10, Article ID 324, 2019.

[6] I. Kim and J. Lee, "Proposed correlation model for groundwater level prediction based on river stage considering changes in hydrological and geological conditions," Journal of Hydrologic Engineering, vol. 24, no. 10, Article ID 4019042, 2019.

[7] F. Li, W. Wei, Y. Zhao, and J. Qiao, "Groundwater depth prediction in a shallow aquifer in north China by a quantile regression model," Hydrogeology Journal, vol. 25, no. 1, pp. 191-202, 2017.

[8] S. Kostić, M. Stojković, I. Guranov, and N. Vasović, "Revealing the background of groundwater level dynamics: contributing factors, complex modeling and engineering applications," Chaos, Solitons \& Fractals, vol. 127, pp. 408421, 2019.

[9] H. K. Moghaddam, H. K. Moghaddam, Z. R. Kivi, M. Bahreinimotlagh, and M. J. Alizadeh, "Developing comparative mathematic models, BN and ANN for forecasting of groundwater levels," Groundwater for Sustainable Development, vol. 9, Article ID 100237, 2019.

[10] J. Tian, C. Li, J. Liu et al., "Groundwater depth prediction using data-driven models with the assistance of gamma test," Sustainability, vol. 8, no. 11, Article ID 1076, 2016.

[11] N. Zhang, C. Xiao, B. Liu, and X. Liang, "Groundwater depth predictions by GSM, RBF, and ANFIS models: a comparative assessment," Arabian Journal of Geosciences, vol. 10, no. 8, Article ID 189, 2017.

[12] M. K. Jha and S. Sahoo, "Efficacy of neural network and genetic algorithm techniques in simulating spatio-temporal fluctuations of groundwater," Hydrological Processes, vol. 29, no. 5, pp. 671-691, 2015.

[13] A. Mirarabi, H. R. Nassery, M. Nakhaei, J. Adamowski, A. H. Akbarzadeh, and F. Alijani, "Evaluation of data-driven models (SVR and ANN) for groundwater-level prediction in confined and unconfined systems," Environmental Earth Sciences, vol. 78, no. 15, Article ID 489, 2019.

[14] T. Guo, S. Song, J. Shi, and J. Li, "Groundwater depth forecasting using configurational entropy spectral analyses with the optimal input," Groundwater, vol. 585, no. 5, pp. 749-758, 2020.

[15] C. Song, X. Zhang, D. Hu, and W. Tuo, "Research on a coupling model for groundwater depth forecasting," Desalination and Water Treatment, vol. 142, pp. 125-135, 2019.

[16] D. Liu, G. Li, Q. Fu et al., "Application of particle swarm optimization and extreme learning machine forecasting models for regional groundwater depth using nonlinear prediction models as preprocessor," Journal of Hydrologic Engineering, vol. 23, no. 12, Article ID 4018052, 2018.

[17] R. W. Healy, Estimating Groundwater Recharge, Cambridge University Press, Cambridge, UK, 2010.

[18] Q. Huang, S. D. Rozelle, R. E. Howitt, and J. E. Wilen, "Optimal allocation of groundwater resources: managing water quantity and quality," Natural Resource Management and Policy, vol. 50, pp. 89-114, 2019.
[19] Y. Zeng, C. Zhao, J. Li, Y. Li, G. Lv, and T. Liu, "Effect of groundwater depth on riparian plant diversity along riversidedesert gradients in the Tarim River," Journal of Plant Ecology, vol. 12, no. 3, pp. 564-573, 2019.

[20] G. Zhang, X. Su, and V. P. Singh, "Modelling groundwaterdependent vegetation index using entropy theory," Ecological Modelling, vol. 416, Article ID 108916, 2020.

[21] I. N. Daliakopoulos, P. Coulibaly, and I. K. Tsanis, "Groundwater level forecasting using artificial neural networks," Journal of Hydrology, vol. 309, no. 1-4, pp. 229-240, 2005.

[22] P.-C. Hsieh, W.-A. Tong, and Y.-C. Wang, "A hybrid approach of artificial neural network and multiple regression to forecast typhoon rainfall and groundwater-level change," Hydrological Sciences Journal, vol. 64, no. 14, pp. 1793-1802, 2019.

[23] D. Julong, "Introduction to grey system theory," Journal of Grey System, vol. 1, no. 1, pp. 1-24, 1989.

[24] L. Wu, S. Liu, L. Yao, S. Yan, and D. Liu, "Grey system model with the fractional order accumulation," Communications in Nonlinear Science and Numerical Simulation, vol. 18, no. 7, pp. 1775-1785, 2013.

[25] S. Bahrami, R.-A. Hooshmand, and M. Parastegari, "Short term electric load forecasting by wavelet transform and grey model improved by PSO (particle swarm optimization) algorithm," Energy, vol. 72, pp. 434-442, 2014.

[26] C.-C. Hsu and C.-Y. Chen, "Applications of improved grey prediction model for power demand forecasting," Energy Conversion and Management, vol. 44, no. 14, pp. 2241-2249, 2003.

[27] Y.-S. Lee and L.-I. Tong, "Forecasting energy consumption using a grey model improved by incorporating genetic programming," Energy Conversion and Management, vol. 52, no. 1, pp. 147-152, 2011.

[28] X. Li, G. Li, and Y. Zhang, "Identifying major factors affecting groundwater change in the North China Plain with grey relational analysis," Water, vol. 6, no. 6, pp. 1581-1600, 2014.

[29] W. Z. Lin, J. A. Fang, X. Xiao, and K. C. Chou, "iDNA-Prot: identification of DNA binding proteins using random forest with grey model," PLoS One, vol. 6, no. 9, Article ID e24756, 2011.

[30] G. Wei, Y. Ling, B. Guo, B. Xiao, and A. V. Vasilakos, "Prediction-based data aggregation in wireless sensor networks: combining grey model and Kalman Filter," Computer Communications, vol. 34, no. 6, pp. 793-802, 2011.

[31] L. Tu and Y. Chen, "An unequal adjacent grey forecasting air pollution urban model," Applied Mathematical Modelling, vol. 99, pp. 260-275, 2021.

[32] L. Wu, S. Liu, Z. Fang, and H. Xu, "Properties of the GM $(1,1)$ with fractional order accumulation," Applied Mathematics and Computation, vol. 252, pp. 287-293, 2015.

[33] Q. Wu, W. Zhou, S. Li, and X. Wu, "Application of grey numerical model to groundwater resource evaluation," Environmental Geology, vol. 47, no. 7, pp. 991-999, 2005.

[34] L. Wu, S. Liu, D. Liu, Z. Fang, and H. Xu, "Modelling and forecasting $\mathrm{CO}_{2}$ emissions in the BRICS (Brazil, Russia, India, China, and South Africa) countries using a novel multivariable grey model," Energy, vol. 79, pp. 489-495, 2015.

[35] L. Wu, S. Liu, L. Yao, and S. Yan, "The effect of sample size on the grey system model," Applied Mathematical Modelling, vol. 37, no. 9, pp. 6577-6583, 2013.

[36] C. Chatfield and M. Yar, "Holt-Winters forecasting: some practical issues," The Statistician, vol. 37, no. 2, pp. 129-140, 1988. 
[37] S. Mirjalili, S. M. Mirjalili, and A. Lewis, "Grey wolf optimizer," Advances in Engineering Software, vol. 69, pp. 46-61, 2014.

[38] Z.-X. Wang, Q. Li, and L.-L. Pei, "Grey forecasting method of quarterly hydropower production in China based on a data grouping approach," Applied Mathematical Modelling, vol. 51, pp. 302-316, 2017.

[39] S. Gaur, B. R. Chahar, and D. Graillot, "Analytic elements method and particle swarm optimization based simulation optimization model for groundwater management," Journal of Hydrology, vol. 402, no. 3-4, pp. 217-227, 2011.

[40] Y. Shi and R. C. Eberhart, "Parameter selection in particle swarm optimization," in Proceedings of the International Conference on Evolutionary Programming, pp. 591-600, San Diego, CA, USA, March 1998.

[41] Z. Li-Ping, Y. Huan-Jun, and H. Shang-Xu, "Optimal choice of parameters for particle swarm optimization," Journal of Zhejiang University - Science, vol. 6, no. 6, pp. 528-534, 2005.

[42] J. Kennedy and R. Eberhart, "Particle swarm optimization," in Proceedings of ICNN'95-International Conference on Neural Networks, Perth, WA, Australia, November 1995.

[43] Y. Fan, H. Li, and G. Miguez-Macho, "Global patterns of groundwater table depth," Science, vol. 339, no. 6122, pp. 940-943, 2013. 